\title{
In memoriam Hartmann F. Stähelin
}

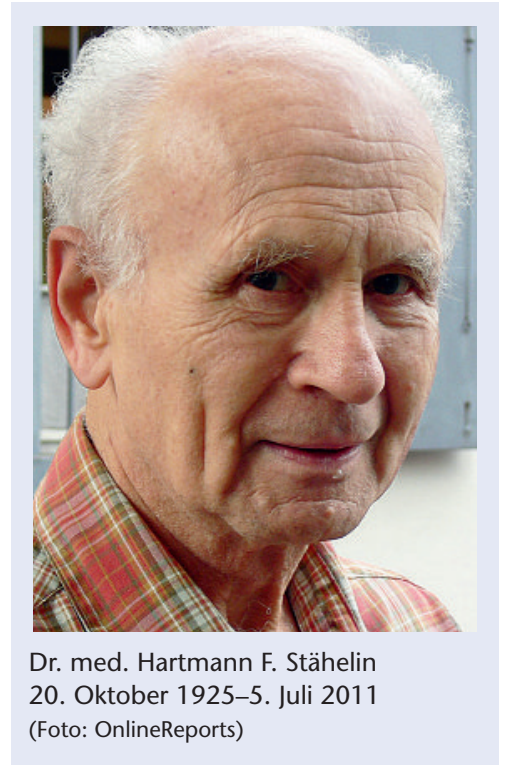

Der Mediziner Hartmann F. Stähelin - der Entdecker der Krebsmedikamente Etoposid, Teniposid und des Immunsuppressivums Cyclosporin - ist am 5. Juli 2011, nur wenige Tage nach einem Sturz auf einer seiner geliebten Velofahrten, an den schweren Verletzungen verstorben. Die Stadt Basel verliert in ihm einen nobelpreiswürdigen Entdecker von heute weltweit gebrauchten lebensrettenden Medikamenten und einen bis zuletzt unermüdlichen, gemeinnützigen Kämpfer für die Redlichkeit in der Forschung.

Das Krebsmedikament Etoposid fand schon früh Aufnahme in die klassische Chemotherapiekombination mit Cisplatin und Bleomycin («BEP») bei der kurativen Behandlung der nicht-seminomatösen Hodentumoren und in vielen anderen bis heute gebräuchlichen onkologischen multimodalen Therapien. Das Cyclosporin revolutionierte bekanntlich die Organtransplantation in den frühen Achtzigerjahren des letzten Jahrhunderts.

Stähelin entstammte einer Medizinerfamile (beide Eltern waren Ärzte), und er studierte nach Abschluss des humanistischen Gymnasiums in Basel, Zürich und Florenz Medizin (1944-1950). Dass dem sportlich vielseitig begabten jungen Stähelin (Bergwandern, Reiten, Skifahren, Tanzen, Basler Juniorenmeister im Tennis) auch in der Wissenschaft schon früh bemerkenswerte «Punktlandungen» gelangen, erstaunt nicht. 1952 absolvierte der mutige 27-Jäh- rige zusammen mit seinem Basler Kollegen Luzius Dettli den ersten Kurs für lebensrettende Fallschirmsprünge im Auftrag der damals neugegründeten Schweizerischen Rettungsflugwacht (heute REGA) bei der Royal Airforce im englischen Abington. Als Assistent am Institut für Mikrobiologe der Universität Basel (1951-1954) beobachtete er im damals neuen Phasenkontrastmikroskop erstmals die Fusion von nackten Protoplasten von Anthraxbakterien, die er in einer aufsehenerregenden Arbeit beschrieben hat, die den Nobelpreisträger Joshua Lederberg auf ihn aufmerksam machte (Publikation 1954). Ein 12-monatiger, vom Schweizerischen Nationalfonds (SNF) unterstützter Auslandsaufenthalt an der Harvard Medical School folgte. Dort begann er auf Anregung des Basler Forschers und Freundes Emanuel Suter im Labor von Manfred L. Karnovsky an einem Projekt über Makrophagen zu arbeiten, das ihn zur Wiederentdeckung des seit 1933 in Vergessenheit geratenen «respiratory burst» führte (Publikation 1956/57).

Während eines Aufenhalts in Boston wurde ein wissenschaftlicher Direktor der Sandoz auf Stähelins aussergewöhnliches Forschertalent aufmerksam. In einem weiteren 6-monatigen - nun von der Sandoz finanzierten - Aufenthalt im Labor des Nobelpreisträgers John F. Enders machte sich Stähelin mit neusten Zellkulturtechniken vertraut, die ihm später als Chef der damals neugegründeten Arbeitsgruppe «Krebs und Immunologie» (1955-1979) zugutekamen.

Was in dieser Zeitspanne an klinisch bedeutsamen Durchbrüchen und Erfolgen auf Stähelins Wirken zurückgeführt werden kann, wurde im Juni 1985 anlässlich der Vergabe des Bristol-Preises der Amerikanischen Gesellschaft für Krebs-Chemotherapie erstmals hervorragend zusammengefasst (Sandorama III/1985). Die offizielle Laudatio ehrt den Entdecker der Krebsmedikamente Teniposid (Oktober 1965) und Etoposid (Oktober 1966) und des Immunsuppressivums Cyclosporin (Januar 1972). Cyclosporin wurde mittels eines von Stähelin eigens im Januar 1970 dafür entwickelten, damals weltweit einmaligen Screening-Systems zur Auffindung von immunsupprimierenden Substanzen ohne Knochemmarkhemmung entdeckt. Der «gezielte», mit manchen Überraschungen gepflasterte Weg, der Stähelin und sein Team vom immunsupprimierenden, aber knochenmarkstoxischen Etoposid über Ovalicin zur Entdeckung von Cyclosporin führte, hat er anlässlich der Vergabe des AACR Bruce F. Cain Awards 1990 an ihn und den Chemiker A. von Wartburg in der Januaraus-
Nachrufs ist am 13.7.2011 in «onlinereports» (www. onlinereports.ch) erschienen. 
gabe von «Cancer Research» 1991 beschrieben. Es verwundert deshalb nicht, dass die Schweizerische Krebsliga Hartmann Stähelin mit dem Schweizer Krebspreis 1991 ehrte. Die eindrücklichen klinischen Resultate der neuartigen Podophyllinderivate Etoposid und Teniposid führten dazu, dass die auf Onkologika spezialisierte US-Pharmafirma Bristol-Myers auf diese Krebsmittel 1978 von der Sandoz AG in Lizenz übernahm. Dass Schicksal wollte es, dass sowohl Etoposid (Vepesid Bristol-Myers) wie auch Cyclosporin (Sandimmun Sandoz) in derselben Novemberwoche des Jahres 1983 von der US-FDA die Marktzulassung erhielten.

Bis zu seiner Pensionierung (1990) setzte sich Stähelin unermüdlich für die Koordination der präklinischen Forschung innerhalb der Sandoz und als Sekretär der Sandoz-Stiftung ein, obschon es ihm äusserst schwerfiel, 1979 seine ihn inspirierende Labortätigkeit durch Firmenentscheid aufgeben zu müssen und sein durch ihn geschultes Laborteam anderweitig eingesetzt zu sehen. Von 1981 bis 2001 brachte er wäh- rend 20 Jahren seine Kenntnisse und Erfahrungen als Vertreter der Stifterfamilie in die Kommission der gemeinnützigen Christoph Merian Stiftung ein. 1977/78 war er Präsident der Naturforschenden Gesellschaft Basel und diente als Delegierter dieser Gesellschaft auch dem Verein zur Förderung der BernoulliEdition als deren Präsident.

Kompetitives Schach (auch gegen den Computer), ein stimulierender Familien- und Freundeskreis, die historische Aufarbeitung seines wissenschaftlichen Lebenswerks und sportliche Betätigungen vieler Art liessen diesen herausragenden Mediziner bis zu seinem unerwarteten Tod am 5. Juli nie ruhen. Hartmann Stähelin-Staehelin hinterlässt seine Ehefrau Irene, zwei Töchter und zwei Söhne und 11 Enkelkinder. Familie, Freunde und Bekannte haben am 12. Juli 2011 in der Theodorskirche in Basel von ihm Abschied genommen.

Dr. med. Rudolf Steiner, Zürich 